\title{
Conduction in Linear Low Density Polyethylene Nanodielectric Materials
}

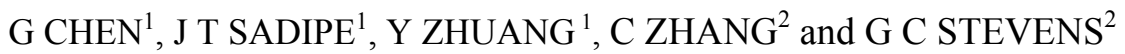 \\ ${ }^{1}$ School of Electronics and Computer Science, University of Southampton, United Kingdom \\ ${ }^{2}$ GnoSys UK Ltd, University of Surrey, United Kingdom \\ *E-mail: gc@ecs.soton.ac.uk
}

\begin{abstract}
Nanodielectrics have been extensively researched in recent years with the expectation that they may enhance electrical performance. In this paper we have studied the electrical conduction processes in linear low density polyethylene (LLDPE) filled with nano alumina particles. The electrical conduction was measured at various applied electric fields. It has been found that the conduction current shows a minimum at a $1 \%$ b.w. concentration nano alumina particles. To aid an understanding of conduction processes in the nanodielectric, differential scanning calorimetry (DSC) was used to analyse the interaction between nano alumina particles and the host matrix. Initial findings suggest that there is an interaction zone around the nano alumina particles. Neighbouring interaction zones are expected to overlap when the percentage of nano alumina particles exceeds $5 \%$ in LLDPE.
\end{abstract}

Keywords: polyethylene insulation; nanodielectric; nano alumina particles; electrical conduction; interaction zone.

\section{INTRODUCTION}

The term nanodielectric refers to a dielectric material which has been filled with nano sized particles in order to modify the properties of the material. It is also referred to as nanocomposite. In such a system, chemically dissimilar components are combined at the nanometer scale, and stronger interactions between the material and nanoparticles produce markedly improved materials with better electrical, mechanical, thermal, and rheological performances. Nanoparticles are usually defined as particles having dimensions smaller than $1000 \mathrm{~nm}$, but more typically of the order of 1 to $100 \mathrm{~nm}$. The field of nanodielectric research is relatively new but a lot of research has been carried out recently incorporating various nanoparticles into existing host dielectric matrices resulting in nanocomposites with improved benefits over conventional micro sized filler systems [1-6]. In reducing the size of the particles, the surface area of the particles increases significantly for the same volume of fillers. This change in the total interfacial area between the filler and the host material means that the mechanisms associated with the interface between two materials is expected to have a significant impact on the macroscopic properties of the composite.
As far as electrical transport properties of all dielectrics are concerned, they are closely associated with charge injection and bulk dynamics. The measurement of charge formation, transportation of nanodielectric materials provides an important tool to understand interaction between nanopartilces and the host material. Our results from space charge measurements for the same samples indicated that there may be an optimal amount ( $\sim 1 \%$ b.w.) of nanoparticles for nanodielectric [7]. Beyond that the dielectric properties deteriorate very rapidly. In the present paper we have studied the electrical conduction processes in linear low density polyethylene loaded with weakly aggregated nano alumina particles. The electrical conduction was measured at various applied electric fields and temperatures. To aid the understanding of conduction processes in these semicrystalline nanodielectrics, differential scanning calorimetry was used to analyse the interaction between nano alumina particles and host matrix.

\section{EXPERIMENTAL DETAILS}

\section{Sample Preparation}

LLDPE used in this study is a commercial linear low density polyethylene from Atofina. It has a density of $0.934 \mathrm{~g} / \mathrm{cm}^{3}$, a melt index of $0.87 \mathrm{~g} / 10 \mathrm{~min}$. Nanoparticles of aluminium oxide from Degussa with a particle size of about $13 \mathrm{~nm}$ was used as filler. This nano scale filler has a nearly spherical shape with a specific surface area of about $100 \mathrm{~m}^{2} / \mathrm{g}$. The filler was dried before use. Polyethylene nanocomposites were prepared by melt mixing at $150^{\circ} \mathrm{C}$ using a Brabender W50EHT mixer with chamber size of $50 \mathrm{~cm}^{3}$ under a nitrogen atmosphere. The mixer has a high shear force, the screw speed was controlled at $60 \mathrm{rpm}$, and the mixing time was $20 \mathrm{~min}$ for each sample. The sheets of mixtures were then extruded by using a Polylab Universal Moulding Machine at $150^{\circ} \mathrm{C}$. The nanocomposites were finally prepared into films with the thickness of $0.2 \mathrm{~mm}$ by hot melt pressing at $150{ }^{\circ} \mathrm{C}$ or $180^{\circ} \mathrm{C}$. The cooling process has a significant influence on the morphological features of the sample which may alter the dielectric characteristics. To keep a consistence morphology the moulded samples were immersed into distilled cool water (quenching). Four types of polyethylene nanocomposite disc films with a 
diameter of $35 \mathrm{~mm}$ were prepared with concentrations of alumina of $0,1,5$, and $10 \mathrm{wt} \%$, respectively. For $10 \%$ the moulding temperature had to be raised to $180^{\circ} \mathrm{C}$ to achieve a uniform sample. Table 1 shows the details of the samples prepared.

Table 1 Sample details

\begin{tabular}{|l|l|l|}
\hline Sample & $\begin{array}{l}\text { Alumina \% by } \\
\text { Weight }\end{array}$ & $\begin{array}{l}\text { Moulding } \\
\text { temperature }\left({ }^{\circ} \mathrm{C}\right)\end{array}$ \\
\hline PE-0 & 0 & 150 \\
\hline PE-1 & 1 & 150 \\
\hline PE-5 & 5 & 150 \\
\hline PE-10 & 10 & 180 \\
\hline
\end{tabular}

\section{Electrical Conduction}

The samples were stressed under various dc electric fields and the electrical current was measured by Keithley 486 picoammeter. Two electrodes (diameter $5 \mathrm{~mm}$ ) system was used in the present study. The electrode system was placed in oven so the desired temperatures can be achieved. The conduction current changes with time under an electric field and takes some time to reach a steady value. The time varies with the electric field applied and temperature. In the present exploratory study we record the current value after 1 hour application of the electric field which is a steady state conduction current.

\section{RESULTS}

Figure 1 shows the $\mathrm{I}-\mathrm{V}$ characteristics at $20^{\circ} \mathrm{C}$ for the samples filled with different amount of alumina. The conduction current increases with the applied voltage as expected. However, the addition of alumina nanoparticles changes the magnitude of conduction current.

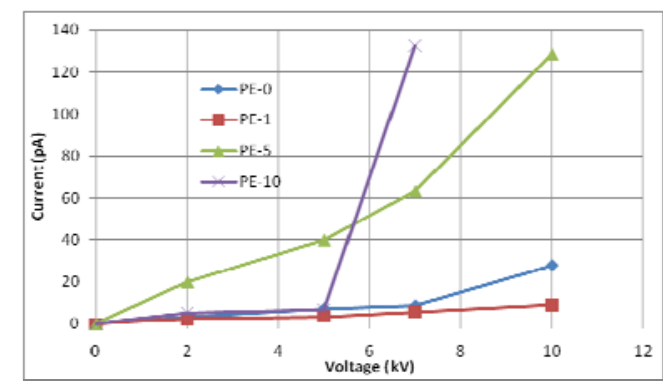

Figure $1 \mathrm{I}-\mathrm{V}$ characteristics in alumina nanopartilces filled LLDPE samples at $20^{\circ} \mathrm{C}$

There are several features which can be seen in this diagram. The first one is the conduction current in the PE-1 sample is lower than the sample without any alumina nanoparticles. This means that the addition of small amount of alumina hinters the movement of charge in the bulk of the material. As the amount of alumina increases the conduction current in the PE-5 increases significantly compared to the PE- 0 . The second feature is the conduction current in the PE-10 sample showing significant reduction at lower voltages but becoming very high once the applied voltage exceeds $5 \mathrm{kV}$. This is unexpected.

Figure 2 illustrates the $\mathrm{I}-\mathrm{V}$ characteristics at $40^{\circ} \mathrm{C}$ for four samples with different amount of alumina nanoparticles. Generally, it shows similar features to those in Figure 1. Increasing temperature leads to higher conduction current for all the samples except PE-10. Additionally, the voltage that causes a rapid increase in the conduction current in PE-10 moves up to $7 \mathrm{kV}$.

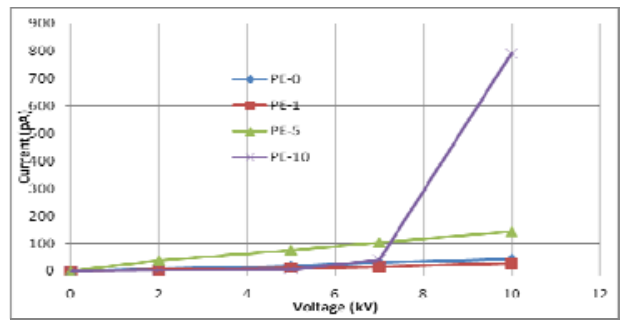

Figure $2 \mathrm{I}-\mathrm{V}$ characteristics in alumina nanopartilces filled LLDPE samples at $40^{\circ} \mathrm{C}$

$\mathrm{I}-\mathrm{V}$ characteristics for all the samples at $60^{\circ} \mathrm{C}$ are shown in Figure 3. The same features as in Figure 2 are observed again. This may demonstrate that different interactions can take place when different amounts of alumina nanoparticles are added.

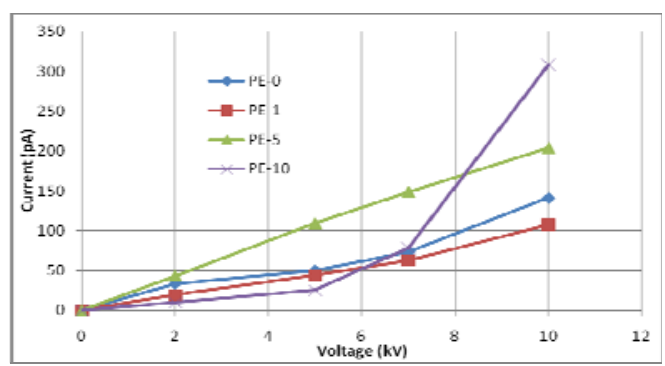

Figure $3 \mathrm{I}-\mathrm{V}$ characteristics in alumina nanopartilces filled LLDPE samples at $60^{\circ} \mathrm{C}$

Based on the above results the relationship between conduction current and temperature in PE-0, PE-1 and PE-5 at different voltages can be derived. The plots show a similar trend and for the simplicity, only the current versus temperature at $5 \mathrm{kV}$ is shown in Figure 4.

The activation energy can be extracted from the current and temperature plot and the average values for PE- 0 , PE-1 and PE-5 are shown in Table 2.

Table 2 Changes in activation energy

\begin{tabular}{|l|l|l|l|}
\hline Sample & PE-0 & PE-1 & PE-5 \\
\hline $\begin{array}{l}\text { Activation } \\
\text { energy (eV) }\end{array}$ & 0.42 & 0.50 & 0.16 \\
\hline
\end{tabular}




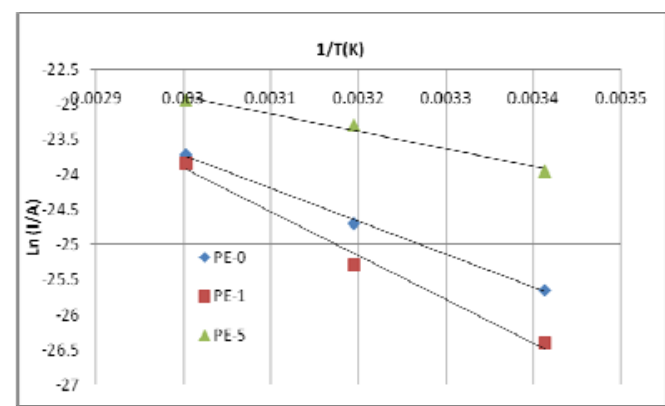

Figure 4 Conduction current versus temperature for samples PE-0, PE-1 and PE-5 at $5 \mathrm{kV}$.

It can be seen that by adding small amount of alumina nanoparticles the activation energy increases. Further addition of alumina nanoparticles results in a decrease in activation energy. All these results are consistent with the conduction current measurements.

The temperature dependence of the conduction current in sample PE-10 is very different from the other three samples as shown in Figures 1 to 3 . Instead of increasing with temperature the conduction current actually decreases.

\section{DISCUSSION}

The addition of nano alumina to LLDPE has altered I $-\mathrm{V}$ characteristics of the material. It has been found the alternation depends heavily on the amount of nano alumina particles. For a small amount such as $1 \%$ by weight, $\mathrm{I}-\mathrm{V}$ characteristics of the material is improved, i.e. less conduction current was observed for the same applied voltage. On the other hand when the amount of nano alumina particles is increased the conduction current increases as shown in Sample PE-5. Calculation of activation energy for the samples reveals that the activation energy changes with the amount of nano alumina particles added which may indicates different interactions between nanoparticles and the host LLDPE. Further increase in nano alumina particles leads to a lower conduction current at lower applied voltage but rapid increase in conduction current at higher applied voltage. More surprisingly, the temperature dependence of conduction current in the PE-10 shows a negative coefficient, indicating a different conduction mechanism from the samples with lower concentration of nano alumina particles.

The alternation in $\mathrm{I}-\mathrm{V}$ characteristics is thought to be related to the interface between nano alumina particles and host LLDPE matrix. The interface may extend to tens of nm but no direct experimental evidence is reported here. For an idealized situation where all the nano spherical shaped particles are assumed to sit on the eight corners of a cube as shown in Figure 5, it is possible to calculate the separation distance between adjacent particles.

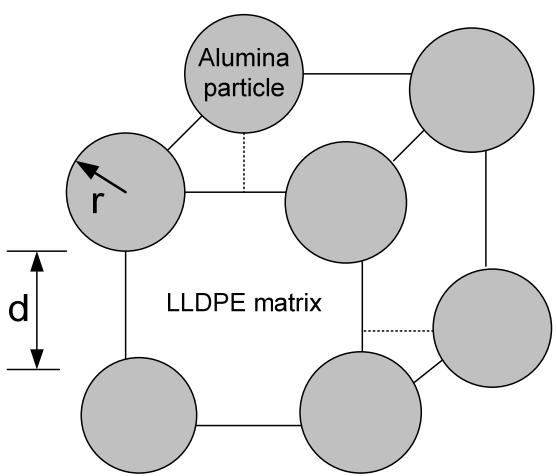

Figure 5 Cubic array of spherical nano alumina particles of radius $r$ and matrix spacing $d$.

$$
d=r\left\{\left[\frac{4 \pi}{3}\left(1+\frac{1-F}{F} \frac{\rho_{f}}{\rho_{m}}\right)\right]^{1 / 3}-2\right\}
$$

$\mathrm{F}$ is the weight fraction of nano alumina particles, $\rho_{\mathrm{f}}=961 \mathrm{~kg} / \mathrm{m}^{3}$ and $\rho_{\mathrm{m}}=920 \mathrm{~kg} / \mathrm{m}^{3}$ are the density of alumina and LLDPE matrix respectively.

Figure 6 shows the separation distance d versus alumina fraction in LLDPE matrix for $\mathrm{r}=6.5 \mathrm{~nm}$. It can be seen that the separation distance varies rapidly for a small percentage of nano alumina particles while it changes slowly for high percentages.

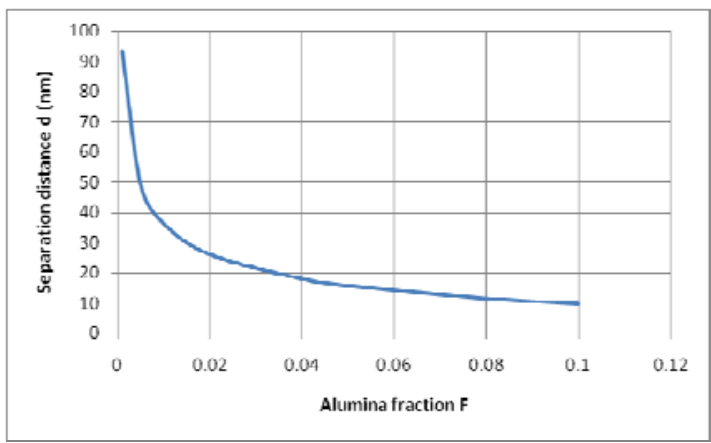

Figure 6 Separation distance between two adjacent nano alumina particles with increasing volume fraction.

To further investigate the results obtained for samples containing different percentage of nano alumina particles, analysis of the melting behavior was carried out using differential scanning calorimetry (DSC). Samples of $\sim 4-5 \mathrm{mg}$ of each of your materials were placed in an aluminium can. The samples started in their molten state $\left(200^{\circ} \mathrm{C}\right)$ and were cooled at $1^{\circ} \mathrm{C}$ per minute to room temperature. The heat flow associated with the crystallisation process is recorded as shown in Figure 7. 


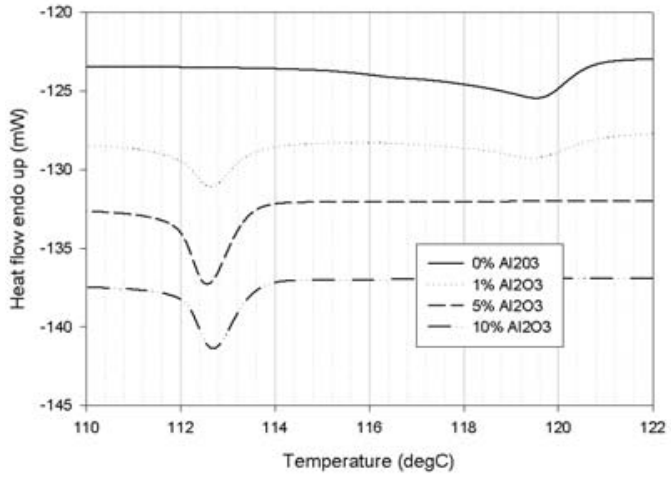

Figure 7 DSC curves for nano alumina filled LLDPE samples

It can be clearly seen that there are two processes, one at just below $120^{\circ} \mathrm{C}$ which occurs in the pure LLDPE and sample filled with $1 \%$ nano alumina particles and one at just above $112^{\circ} \mathrm{C}$ which appears in all the filled samples. It is interesting to notice that only the sample PE-1 has both of these features. This may be seen as direct evidence for an interaction zone around the nanoparticles - ie a region where the LLDPE behaves completely differently than in the absence of nano alumina fillers. In the $5 \%$ and $10 \%$ cases, the sample is then composed entirely of this interface region. It is believed that the confrontation of nano alumina particles and polymer matrix with dissimilar structures introduces a transition or interaction zone through which the properties change from those of the filler particle to the bulk polymer matrix. It is also possible that the interaction zone may behave completely differently. A schematic diagram is shown in Figure 8 to represent the interaction zone and overlap in the presence of higher percentage of nano alumina particles. Based on the idealised model shown in Figure 5, the radius of the interaction zone can be estimated from the separation distance shown in Figure 6. For 5\%, the separation distance is around $15.8 \mathrm{~nm}$, so the radius of the interaction zone is around $14.4 \mathrm{~nm}$.

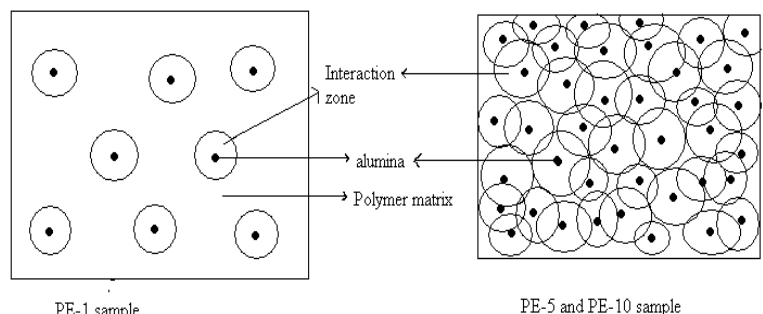

Figure 8 Schematic diagram of interaction zone around nano alumina particles.

In the case of PE-10, the overlap of the interaction zone is so strong and the charge tunneling dominates charge transportation process. The tunneling process generally shows strong electric field dependence. At a lower applied voltage the electric field is not higher enough resulting in a low conduction current. Once the electric field due to the applied voltage is sufficiently high the conduction current will increase rapidly with the voltage as shown in Figures 1 to 3. When temperature increases the sample will expand. The expansion of the sample will make the effective distance between two nano alumina particles larger. This will subsequently reduce the tunneling efficiency leading to a lower conduction current when the temperature increases. This explains why the conduction current in PE-10 shows a negative temperature coefficient. If one accepts the multizone model proposed by Tanaka [8], then conduction the current characteristics for PE-5 and PE-10 may indicate that each zone behaves differently.

\section{CONCLUSIONS}

The conduction current in PE-1 is the lowest, indicating that there is an optimal amount of nano alumina particles which is possibly close to $1 \%$ b.w.. This is consistent with previous space charge measurements. In such a case, individual features of interaction zone and host matrix are coexistent. The conduction current starts to increase in PE-5 and DSC results reveal that the feature of the interaction zone completely takes over in this case. The estimated radius of the interaction zone is around $14 \mathrm{~nm}$. Further increase in nano alumina particles leads to a different conduction process probably tunneling - suggesting that the different zones behave differently prior to and after zone overlap.

\section{Acknowledgement}

Authors thank Dr. Chris Green for running DSC experiments and stimulating discussion.

\section{REFERENCES}

[1] T. J Lewis "Nanometric Dielectrics" IEEE Tran. on Diel. and Elec. Ins. Vol. 1, pp. 812-825, 1994

[2] Y. Cao, P. C. Irwin and K. Younsi "The Future of Nanodielectrics in the Electrical Power Industry" IEEE Tran. on Diel. and Elec. Ins. Vol. 11, pp. 797-807, 2004.

[3] T. Tanaka "Dielectric Nanocomposites with Insulating Properties" IEEE Tran. on Diel. and Elec. Ins. Vol. 12, pp. 914-928, 2005.

[4] J. K. Nelson and Y. Hu "Candidate Mechanisms Responsible for Property Changes in Dielectric Nanocomposites" IEEE Conf. on Prop. \& Appl. of Diel. Mat., pp. 150-154, 2006.

[5] M. F. Frechette, M. L. Trudeau, H. D. Alamdari and S. Boily "Introductory Remarks on Nanodielectrics" IEEE Tran. on Diel. and Elec. Ins. Vol. 11, pp.808-818, 2005.

[6] C. Zhang and G.C. Stevens, "The Dielectric Response of Polar and Non-polar Nanodielectrics”, IEEE TDEI, Vol. 15, pp. 606 - 617, 2008 .

[7] G. Chen, C. Zhang and G.C Stevens, "Space charge in LLDPE loaded with nanoparticles" CEIDP, Vancouver, Canada, pp. 275 278, 2007.

[8] T. Tanaka "Interpretation of Several Key Phenomena Peculiar to Nano Dielectrics in terms of a Multi-core Model" CEIDP, Kansas City, Mo, USA, pp. 298-301, 2006. 